\title{
Recolección de hongos comestibles silvestres y estrategias para el reconocimiento de especies tóxicas entre los tsotsiles de Chamula, Chiapas, México
}

\author{
Collection of wild edible fungi and strategies for the recognition of toxic \\ species among the tsotsiles of Chamula, Chiapas, Mexico
}

\section{Felipe Ruan-Soto}

UNAM, Becario del Programa de Becas Posdoctorales en la UNAM, Centro de Investigaciones Multidisciplinarias sobre Chiapas y la Frontera Sur. Calle María Adelina Flores No. 34-A, Barrio Guadalupe. CP 29230, San Cristóbal de Las Casas, Chiapas, México.

\section{RESUMEN}

Antecedentes: En los Altos de Chiapas, México, los hongos comestibles silvestres son un recurso apreciado, sobre todo por la población indígena tsotsil y tseltal. Sin embargo, en años recientes las intoxicaciones mortales por consumo de hongos han sido frecuentes.

Objetivos: Se describen y analizan las prácticas de recolecta que tienen los tsotsiles de Chamula, cómo han ido cambiando con el tiempo, así como las estrategias para identificar especies comestibles y tóxicas.

Método: El estudio se llevó a cabo en Chamula, Chiapas, zona montañosa de bosques templados con población maya tsotsil. Partiendo de un enfoque etnográfico y a través de métodos cualitativos, se realizaron entrevistas no estructuradas y semiestructuradas, así como recolectas de ejemplares fúngicos.

Resultados: Antiguamente la recolecta de hongos era una práctica común. Alrededor de 21 especies se enunciaron como comestibles, la mayoría ectomicorrizógenas. Actualmente existe la percepción de que la recolecta no es una práctica frecuente por distintos factores como la disminución de la cobertura forestal o el abandono de estas prácticas de aprovechamiento. Se mencionaron distintas estrategias para reconocer los hongos comestibles.

Conclusiones: Aunque la recolecta de hongos subsiste entre los tsotsiles de Chamula, existe un riesgo de salud por la pérdida de conocimientos tradicionales.

Palabras clave: etnomicología, etnobiología, conocimientos micológicos tradicionales, Altos de Chiapas, productos forestales no maderables.

\section{ABSTRACT}

Background: In the Highlands of Chiapas, Mexico, wild edible mushrooms are a priced resource, especially among Tsotsil and Tseltal indigenous peoples. However, in recent years deathly intoxications by mushrooms have been frequent.

Objectives: The collection practices of the Tsotsil of Chamula are described and analyzed, along with the ways in which they have changed over time and the strategies to identify toxic and edible species.

Method: This study was carried out in Chamula, Chiapas, a mountainous zone with temperate forest and a Tsotsil Maya population. With an ethnographic approach and using qualitative methods, non-structured and semi-structured interviews were carried out along with collection of fungal specimens.

Results: In the past, mushroom collection was a common practice. Around 21 species were cited as edible, most of them ectomycorrhizal. Currently, collection is not perceived as a frequent practice due to several factors, including forest reduction and the abandonment of these use practices. Several strategies to recognize edible species were mentioned.

Conclusions: Although mushroom collecting persists among Chamula Tsotsils, there is a risk to their health because of loss of traditional knowledge.

Keywords: Ethnomycology, Ethnobiology, Traditional Mycological Knowledge, Highlands of Chiapas, Non-timber Forest Products.

\section{ARTICLE HISTORY}

Received 02 April 2018 / Accepted 26 July 2018

On line 02 November 2018

\section{INTRODUCCIÓN}

Un alto porcentaje de la población, sobre todo en regiones pobres, vive cerca de los bosques y es en

\section{CORRESPONDING AUTHOR}

I Felipe Ruan-Soto, ruansoto@yahoo.com.mx

ORCID: 0000-0002-2476-027X

estos espacios donde obtiene una considerable cantidad de alimentos. Aunque hoy en día nadie subsiste exclusivamente de la recolección, las especies comestibles silvestres proporcionan una parte importante de 
la alimentación, jugando un papel importante en las dietas de grupos étnicos en todo el mundo (Colfer et al., 2006; Rapoport y Ladio, 1999). Dentro de este grupo de alimentos, los hongos silvestres son un recurso recolectado intensivamente en muchas regiones de México y el mundo durante la época de lluvias tanto para el autoconsumo como para su comercialización, ya que constituyen un importante complemento de proteína en la dieta y una fuente de ingresos monetarios relativamente altos (Boa, 2004; Garibay-Orijel y Ruan-Soto, 2014). Particularmente en Los Altos de Chiapas, México, algunos estudios han dado cuenta de las especies de hongos comestibles silvestres que son consumidas, sus nombres y sistemática local y algunos aspectos relacionados con cómo perciben a estos organismos, sobre todo con pueblos tseltales (Alvarado-Rodríguez, 2010; Lampman, 2007; Ruan-Soto et al., 2013; Shepard et al., 2008).

Sin embargo, en los bosques también existen especies de hongos que contienen compuestos tóxicos cuya ingesta produce efectos nocivos en la salud llamados micetismos. Estos pueden ir desde una leve intoxicación que provoque malestares gastrointestinales hasta envenenamientos cuyo desenlace es la muerte (Lincoff y Mitchel, 1977; Ruiz et al., 1999). En el mundo, se estima que deben existir entre 100 y 150 especies tóxicas (Eren et al., 2010; Graeme, 2014) y tan solo 18 especies mortales (Turner y Szczawinski, 1997). En México, pese a que en el pasado se consideraba que los casos de intoxicación por consumo de hongos eran excepcionales, y localizados principalmente en el centro del país, desde principios del siglo XXI se han registrado más de 200 casos en al menos siete estados (Ramírez-Terrazo, 2017). En Chiapas, a partir de 2005 se ha hecho evidente la ocurrencia de intoxicaciones por consumo de hongos silvestres (Ruan-Soto et al., 2012), reportándose 85 casos, 31 de ellos letales (Vilchis, 2015) en la región de Los Altos de Chiapas, particularmente en el municipio de Chamula.

Diferentes estudios etnomicológicos han dado cuenta de que, a lo largo del tiempo, la gente de poblados micófagos ha construido conocimientos específicos para reconocer las especies de hongos que pueden ser consumidas (Montoya et al., 2007; Ramírez-Terrazo et al., 2014). Sin embargo, la etnomicología y la etnobiología en general, también ha testificado la pérdida de estos conocimientos por diversos factores que acarrea la modernidad y el cambio cultural (Valencia,
2006; Saynes-Vázquez et al., 2013). Esto puede desencadenar ya sea una tendencia al abandono de los recursos fúngicos o recolectas no seguras que pudieran poner en peligro la salud de los consumidores de especies silvestres.

En el presente estudio describimos y analizamos las prácticas de recolecta que tienen los tsotsiles de San Juan Chamula, Chiapas, cómo han ido cambiando estas con el paso de los años, las estrategias que tienen para identificar las especies comestibles de aquellas que pudieran ser consideradas como tóxicas, así como las especies comestibles y tóxicas más importantes para la gente entrevistada. Con este trabajo, se pretende abordar algunos aspectos de la problemática de salud pública que ha aquejado a esta población en los últimos años, contextualizando algunas de sus facetas.

\section{MATERIALES Y MÉTODOS}

\section{Área de estudio}

El municipio de San Juan Chamula se encuentra ubicado en la región de los Altos de Chiapas, en la parte central del Estado, al sureste de México, entre la latitud $16^{\circ} 47^{\prime}$ norte y la longitud de $92^{\circ} 41^{\prime}$ oeste (Figura 1). Cuenta con una extensión de $345665 \mathrm{~km}^{2}$ y una altitud media de 2,260 m s.n.m. El clima predominante es templado subhúmedo $C(w 2)$. El terreno en su gran mayoría es montañoso y accidentado. En cuanto a la precipitación, el municipio cuenta con un régimen de 1,024 mm anuales y una temporada de lluvias que se extiende del mes de mayo hasta noviembre aproximadamente. La temperatura media anual es de $18{ }^{\circ} \mathrm{C}$. (INAFED, 2005). La vegetación característica es de bosques templados con presencia de pino (Pinus spp.) y encino (Quercus spp.) en una área boscosa que se extiende por alrededor de 7,000 ha y donde se han registrado alrededor de 6,000 especies de plantas vasculares (Breedlove, 1981; Lampman, 2007). El resto del territorio lo componen áreas de vegetación secundaria y zonas de cultivo. Chamula tiene una población de 87,332 personas hablantes del Tsotsil, una lengua mayense del grupo tseltalano (INEGI 2015). La cabecera municipal es el asentamiento más grande, sin embargo existen alrededor de 30 comunidades más denominadas parajes. En el municipio las actividades económicas primarias son de gran importancia, seguidas del comercio y el turismo. El cultivo de la milpa es el eje no solo económico sino cul- 


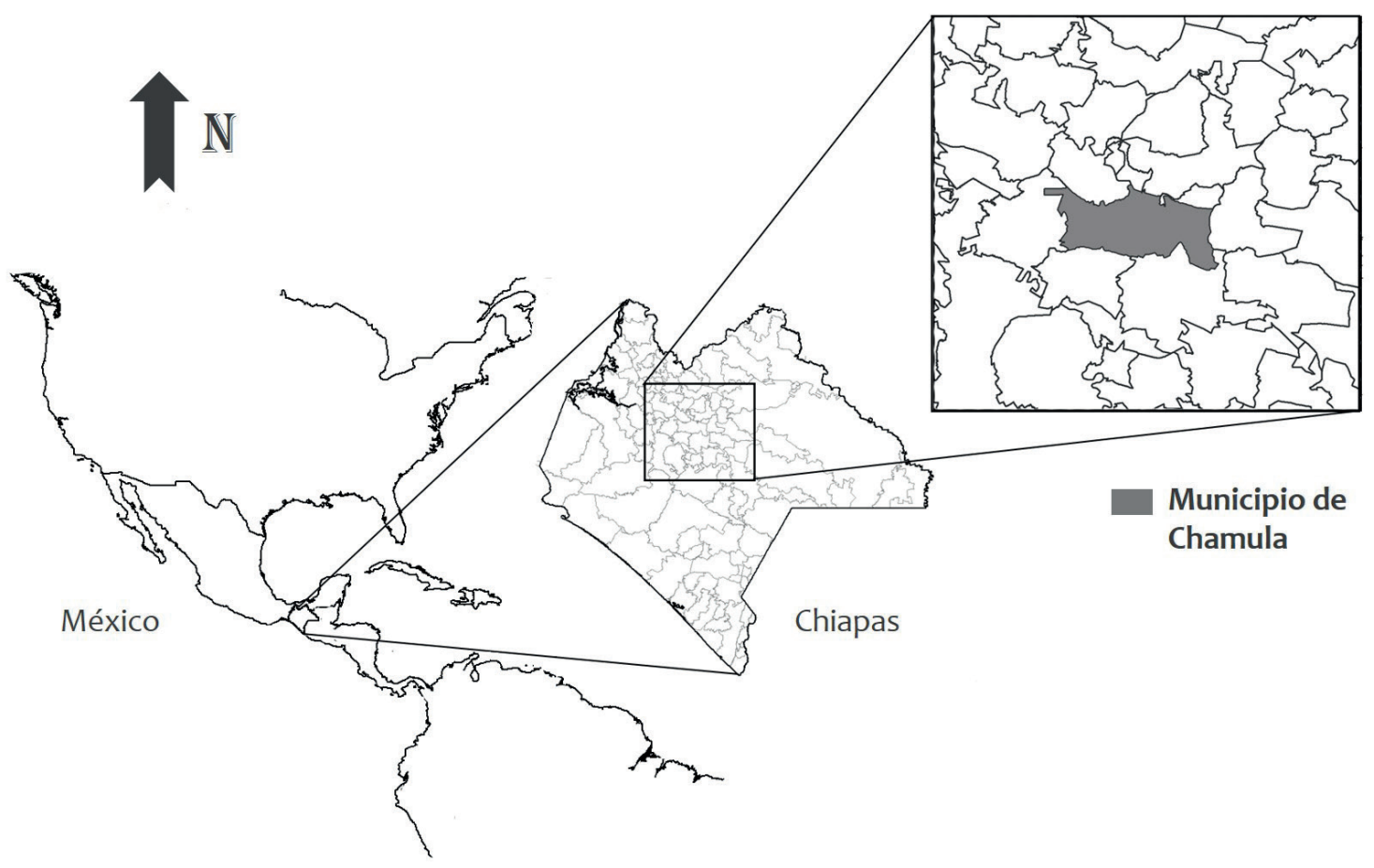

Figura 1. Área de estudio. Municipio de Chamula, Chiapas, México.

tural y social de los tsotsiles de Chamula; a través de ella reproducen aspectos básicos de su cosmovisión (Mariaca et al., 2007). Asimismo, el pastoreo de borregos es una actividad de suma importancia económica, social y cultural, en la cual participan sobre todo las mujeres y los niños (Perezgrovas y Castro, 2000). La recolecta de leña, una actividad también preferentemente femenina, es una actividad sumamente importante por ser la principal (y frecuentemente la única) fuente energética en la unidad doméstica (Soares, 2006). Recientemente la migración de la población masculina como trabajadores asalariados a los núcleos urbanos como a la ciudad vecina San Cristóbal de Las Casas, a los estados de la península de Yucatán, e inclusive a Estados Unidos, es una constante en el municipio y en la región.

\section{Obtención de datos}

La presente investigación, en su fase de campo, se desarrolló entre los meses de mayo y septiembre de 2017. Previamente se solicitó un consentimiento a las autoridades políticas y tradicionales municipales y de algunos parajes para realizar entrevistas a los pobladores, para realizar recolectas de material fúngico y para poder publicar los resultados e imágenes de esta investigación. Todo el trabajo se llevó a cabo siguiendo los lineamientos del código de ética de la Sociedad Latinoamericana de Etnobiología (Cano et al., 2016).

Partiendo de un enfoque etnográfico y a través de métodos cualitativos, se realizaron entrevistas no estructuradas y semiestructuradas de acuerdo a lo propuesto por Bernard (1995) a 34 personas, que acostumbran o acostumbraron recolectar hongos en los bosques de manera frecuente. Todos los entrevistados son tsotsiles, mayores de edad y seleccionados a través de la herramienta de la bola de nieve (Sandoval, 2002). Estas personas son procedentes de los parajes de Yalbante', Yaltsunun, Yaltem,Suyul, Yut bax I, Nichen, Lhomo, Chikviltenal, Jomalo, Las ollas, Jtzav, Laguna petej, Joltojtik, Jolpajaltón, Yolonbe y la cabecera municipal. Debido a que muchas de las personas entrevistadas optaron por guardar el anonimato, en este manuscrito no se menciona ni el nombre ni el paraje de procedencia específico de los colaboradores. Las entrevistas fueron realizadas en español por el autor y traducidas con ayuda de una colaboradora intérprete; giraron en torno a las prácticas de recolección, conocimientos etnoecológicos y de sistemática local, consumo actual de especies comestibles, formas de preparación de los hongos comestibles, transmisión de conocimientos y las formas para diferenciar hongos comestibles de 
tóxicos. Los datos se analizaron de manera cualitativa mediante un contraste de dichas categorías (Sandoval, 2002). Para reconocer a qué especie corresponden los nombres tsostiles de los hongos vertidos en las entrevistas, se realizaron recolectas de ejemplares siguiendo lo establecido por Cifuentes et al. (1986) para su extracción, descripción de las características morfológicas macroscópicas, fotografiado, deshidratado, revisión microscópica e identificación taxonómica.

\section{RESULTADOS Y DISCUSIÓN}

\section{La recolección de hongos en la tradición de Chamula}

En el municipio de Chamula, al interior de sus distintos parajes, la práctica de recolección de hongos comestibles y su consumo no es un tema ajeno. Los entrevistados relatan cómo desde que tienen memoria, salir a buscar hongos era una práctica común y la llevaba a cabo una buena parte de la población en los parajes. Antiguamente, al existir una mayor cobertura forestal, sobre todo de bosques de -tulan- (Quercus spp.), no tenían que caminar mucho para encontrarlos. Esta actividad la llevaban a cabo sobre todo mujeres y niños cuando llevaban a pastorear a sus borregos y cuando salían en búsqueda de leña. A diferencia de este último recurso cuyo acceso es restringido a predios específicos, los hongos pueden ser recolectados de cualquier lugar sin restricciones aun cuando los predios no sean propiedad de quien recolecte. Las personas salían por la mañana (4 ○ 5 am aproximadamente) a lugares específicos donde ya sabían que encontrarían las especies comestibles. Incluso, muchas familias recolectaban para llevarlos a vender a la ciudad de San Cristóbal de Las Casas.

"Cuando era niño sí cortaba hongos, allá por Joltojtik, no había casas, salía bastante del jonguillo y el yuy y lo cortábamos, cuando era puro campo. Iba con la familia, íbamos por leña y si veíamos los cortábamos" (Anónimo, julio de 2017).

"Yo crecí bien rara, ¡buscaba siempre hongos! Porque mi madre me crió así, iba a pastorear, salía en la madrugada, a las cinco de la mañana iba por ahí, entonces ya levantado el sol como a las siete, ya regresaba, después iba de regreso con mis borregos y mi vaca, buscaba otros y los encontraba, sí, es que ya es mi costumbre. Ah sí, de hecho la gente dice eso, -¿dónde los encuentras?- dicen, que van allá, pero que no los ven, ahí lo dejan, aunque ellos pasen primero pero yo los encuentro donde pasaron, así es" (Anónimo, agosto de 2017).

En distintos estudios etnomicológicos a lo largo del mundo, entre diferentes grupos culturales, se ha observado que el acceso libre a los recursos fúngicos es un patrón, ya que al ser percibidos como un bien sin una propiedad predeterminada, pueden ser recolectados en cualquier sitio sin restricción (Härkönen et al., 1993; Mariaca et al., 2001,). Asimismo, es de resaltar el papel de las mujeres en la recolecta de los hongos silvestres. Garibay-Orijel et al. (2012) afirman que en muchas regiones del mundo, las mujeres son las principales recolectoras de hongos, además de ser poseedoras de un vasto conocimiento etnoecológico y etnobiológico al respecto de las especies fúngicas y tener un papel fundamental en la transmisión de estos conocimientos.

\section{Especies comestibles y tóxicas}

Las especies de macromicetos comestibles recolectados más mencionadas en las entrevistas son el -yuy(Amanita hayalyuy), el -k'antsu- (Amanita jacksonii), el -chechev- (Armillaria mellea) el -moni'- (Agaricus spp.) el -sekub t'ul- (Boletus spp.), el -pan chuch- (Suillus spp.) el -yisimchij- (Ramaria spp.) y el -chakat'ob- (Hypomyces lactifluorum) (Figura 2). En la Tabla 1 se observan los etnotaxa que la gente recolectaba en el municipio de Chamula, sus nombres en tsotsil de Chamula y su correspondencia con especies biológicas. Cabe señalar que en dicha Tabla aparecen especies correspondientes a algunas recolectas realizadas en la zona de estudio identificadas por los entrevistados como especies comestibles, sin embargo, no se pude asegurar que dichas especies sean las únicas que corresponden a los etnotaxa mencionados. Al parecer algunos etnotaxa pueden contener además de las especies mencionadas en la Tabla 1, a otras no identificadas. Por ejemplo el etnotaxón, -moni'- puede corresponder a diferentes especies del género Agaricus. Así mismo los etnotaxa -sekub t’ul, -pan chuch-, -yisim chij-, -vuch tuluk-, -nukul chikin- y -yok wakax- corresponden a distintas especies de los géneros Boletus, Suillus, Ramaria, Daldinia, Lentinus e Hydnum respectivamente. En otros estudios etnomicológicos desarrollados en el centro, sur y occidente de México (Garibay-Orijel et al., 2007; Burrola-Aguilar et al., 2012; Montoya et al., 2012; Alonso Aguilar et al., 2014; Gómez-Reyes, 2014), especies de los géneros Amanita, Boletus, Agaricus y Ramaria, también son de 


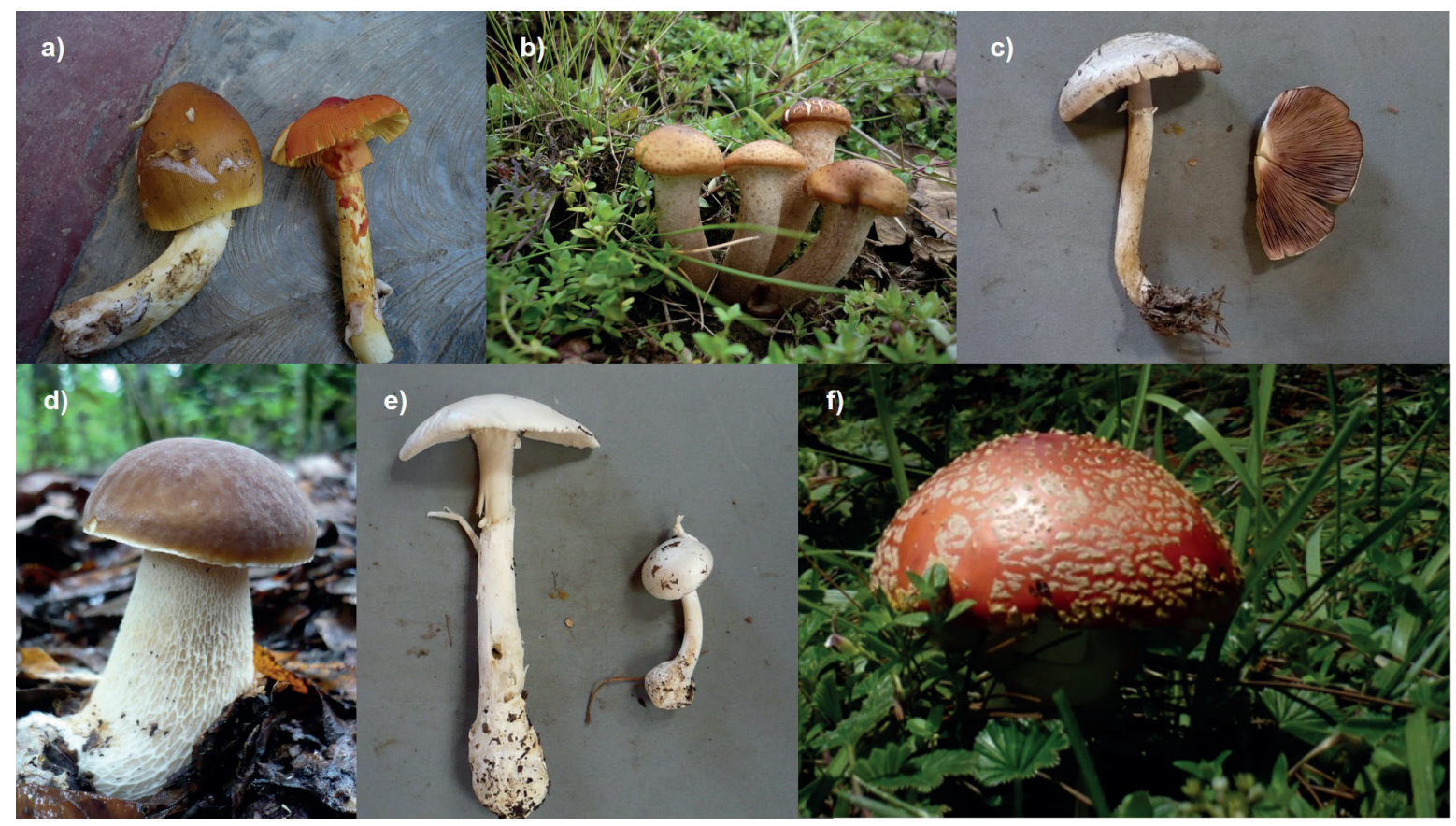

Figura 2. Algunos ejemplos de especies comestibles y tóxicas de Chamula, Chiapas, México. a) Amanita hayalyuy y A. jacksonii; b) Armillaria mellea; c) Agaricus sp.; d) Boletus pinophilus; e) Amanita bisporigera; f) Amanita muscaria.

las especies más mencionadas. Asimismo, también son considerados de los géneros de hongos comestibles más importantes en el mundo (Boa, 2005).

De las 21 especies registradas, dos terceras partes son ectomicorrizógenas, en tanto que solamente siete especies son saprobias. Este es un patrón que se ha observado en otros estudios desarrollados en zonas templadas (Garibay-Orijel et al., 2007; Burrola-Aguilar et al., 2012; Montoya et al., 2012), donde al parecer la gente prefiere especies de mayor tamaño y consistencia carnosa (Härkönen, 1998). Sin embargo, a diferencia de otras zonas templadas, existen especies, sobre todo saprobias, de consumo más moderado como Schizophyllum commune, Lentinus strigellus o Pleurotus djamor, pero que son de gran importancia en tierras bajas tropicales de Mesoamérica (Ruan-Soto y García Santiago, 2013) y la Amazonía (Vasco-Palacios et al., 2008).

\section{La recolecta de hongos en la actualidad}

Actualmente, la gente tiene la percepción de que la recolección de hongos es una práctica que ya no es tan frecuente, debido a que no existen tantos bosques o sitios propicios para su desarrollo, producto de la tala - la disposición de estas tierras para la ubicación de casas habitación o zonas de cultivo. En consecuencia, los hongos comestibles ahora son un recurso considerado escaso. Hoy en día la gente que sale, solamente en ocasiones llega a encontrar algunos esporomas de ciertas especies, o tiene que caminar largas distancias para llegar a sitios con una cobertura forestal mayor que permita el desarrollo de los hongos de mayor importancia. Estudios de deforestación y fragmentación en los Altos de Chiapas han mostrado cómo la cobertura vegetal original de bosques de pino, encino y bosques de niebla, han reducido drásticamente su extensión con tasas de deforestación que van del $1 \%$ al $5 \%$ anual, perdiendo en tan solo una década (19902000) alrededor del 40 \% del bosque (Cayuela, 2006). Los macromicetos, sobre todo los ectomicorrizógenos, dependen completamente de la presencia de la cobertura vegetal arbórea; al disminuir esta, es lógico pensar una disminución de la riqueza y la abundancia de esporomas por pérdida de hábitat.

La gente considera multifactorial la disminución percibida de hongos; de tal modo, da otras explicaciones para ello además de la disminución de la cobertura forestal. Una de ellas es la presencia creciente de agroquímicos en la tierra, producto de las prácticas modernas de siembra tecnificadas. Otra causa atribuida es la erupción del volcán Chichonal en 1982, la cual cubrió de cenizas algunas laderas de montañas que daban la cara al nororiente.

"Antes había más hongo por que no se echaba tanto líquido en la tierra" (Anónimo, septiembre de 2017). 
RUAN SOTO, F. RECOLECCIÓN DE HONGOS COMESTIBLES...

TABLA 1. Especies de hongos comestibles y tóxicos recolectados en el municipio de Chamula, Chiapas, México

\begin{tabular}{|c|c|c|c|}
\hline Especie comestible & $\begin{array}{c}\text { Nombre tsotsil de } \\
\text { Chamula }\end{array}$ & Especie tóxica & Nombre tsotsil de Chamula \\
\hline Amanita hayalyuy Arora \& G.H. Shepard & Yuy & Suillellus luridus (Schaeff.) Murrill & Sekub t'ul jmilvanej \\
\hline Amanita cf. jacksonii Pomerl. & K'antsu & Amanita muscaria (L.) Lam. & Yuy chauk \\
\hline Armillaria mellea (Vahl) P. Kumm. & Chechev & Russula emetica (Schaeff.) Pers. & Sat chij \\
\hline Agaricus sp. & Moni' & $\begin{array}{c}\text { Amanita arocheae Tulloss, Ovrebo \& } \\
\text { Halling }\end{array}$ & Cholchol be jmilvanej \\
\hline Boletus pinophilus Pilát \& Dermek & Sekub t'ul & Hypholoma fasciculare (Huds.) P. Kumm. & K'anal chechev jmilvanej \\
\hline Suillus tomentosus (Kauffman) Singer & Pan chuch & Scleroderma areolatum Ehrenb. & Sat pukuj \\
\hline Ramaria sp. & Yisimchij & Amanita flavoconia G.F. Atk. & Yuy jmilvanej \\
\hline Laccaria laccata (Scop.) Cooke & Kavixtoj & Amanita virosa Bertill. & Sakil yuy \\
\hline Laccaria amethystina Cooke & Yaxal kavixtoj & Amanita aff. phalloides (Vaill. ex Fr.) Link & Vixil yuy \\
\hline Hypomyces lactifluorum (Schwein.) Tul. \& C. Tul. & Chakat'ob & Amanita bisporigera G.F. Atk. & Sakil yuy \\
\hline Neolentinus lepideus (Fr.) Redhead \& Ginns & Taj chuch & Galerina marginata (Batsch) Kühner & Chechev jmilvanej \\
\hline Pleurotus djamor (Rumph. ex Fr.) Boedijn & Sakitaj & Ramaria aff. formosa (Pers.) Quél & Yisim chij jmilvanej \\
\hline Lepista sp. & Checheval mail & Agaricus cf. xanthodermus Genev. & Moni' jmilvanej \\
\hline Lactarius complex. deliciosus (L.) Gray & K'anal manayok & & \\
\hline Cantharellus complex. cibarius Fr. & Xmanayok & & \\
\hline Lactarius indigo (Schwein.) Fr. & Yaxal manayok & & \\
\hline Daldinia loculatoides Wollw. \& M. Stadler & Vuch tuluk & & \\
\hline Amanita vaginata (Bull.) Lam. & Ik' al yuy & & \\
\hline Schizophyllum commune Fr. & Usum & & \\
\hline Lentinus strigellus Berk. & Nukul chikin & & \\
\hline Hydnum repandum L. & Yok wakax & & \\
\hline
\end{tabular}

"Antes sí había mucho, en una vereda salía cantidad. Ahora ya no hay por la ceniza que echó el Chichonal; acabó con los hongos, ya no hay nada" (Anónimo, septiembre de 2017).

En el caso de los agroquímicos, es bien conocido que éstos y sus residuos pueden producir cambios en las comunidades fúngicas de suelos agrícolas sobre todo para los micromicetos (Vázquez, 2015). En cuanto a los macromicetos, se ha observado que con disturbios agudos, las especies ectomicorrizógenas decaen a menos de un $40 \%$ de la composición original, mientras que las especies saprobias lignícolas aumentan más de 40 \% (Dighton, 2003). En Chamula, el uso frecuente e intensivo de agroquímicos, está documentado por diferentes investigadores (por ejemplo Bernardino et al., 2016), convirtiéndose en una práctica común.

En el caso de las cenizas volcánicas, se ha observado cómo después de la caída de cenizas e incluso de material piroclástico, aparecen fructificaciones de especies de hongos fenicoides, sobre todo ascomicetos, debido principalmente a la alteración de la acidez u otras características químicas del suelo. Posteriormente la comunidad fúngica va cambiando, restaurándose la micobiota normal del ambiente alterado, sobre todo aquellas especies de basidiomicetos (Carpenter et al., 1987). Las especies de macromicetos micorrizógenos que sobrevivan estarán directamente relacionadas con las especies vegetales que hayan sobrevivido.

Tanto en el caso de las cenizas como en el del uso de agroquímicos, al ser especies ectomicorrizógenas las de mayor importancia y las más buscadas por los recolectores de Chamula, existe la posibilidad de que efectivamente exista una disminución en la producción de esporomas en tiempos recientes por los cambios en las características químicas del suelo, sin embargo, no existen estudios ecológicos concluyentes que apoyen de manera definitiva esta teoría.

Para otros entrevistados, la baja abundancia reciente no solo tiene que ver con aspectos ecológicos, sino 
también con aspectos sobrenaturales relacionadas con su cosmovisión. Para muchos entrevistados, el que no se encuentre tanta abundancia de hongos comestibles como antaño, es debido a que estos "cambiaron de lugar":

"Este año no encontré, parece que se cambiaron de lugar. Antes recolectaban mucho, había mucho que no lo terminaban de juntar y quedaba pudriéndose en el bosque, por eso ya no hay creo. No supimos cuidar los hongos. Se desperdiciaba y luego ya no hay. Solo recolectaban a los jóvenes y dejaban a los maduros. Por eso desaparecieron los hongos. Si hubiera juntado todos, todavía habría hongos. Es que aquí el costumbre es que hay que cuidar a los alimentos, si se desperdicia viene el castigo y es que ya no van a mandar más" (Anónimo, septiembre de 2017).

Para los tsotsiles de Chamula, los alimentos deben de ser cuidados, ya que si se desperdician, puede acaecer un castigo de orden divino que traiga escasez de alimentos (Mariaca et al., 2007). Para los entrevistados, en el pasado los recolectores no cuidaron los hongos, ya que al haber tanta abundancia, no se recolectaron todos desperdiciándose en el bosque. Esto trajo como consecuencia que se cambiaran de lugar y aparecieran otros hongos diferentes cuya comestibilidad se desconoce.

Aunado a la disminución percibida de la abundancia de esporomas comestibles, entre los entrevistados también existe la percepción de que la gente ya no va tan frecuentemente al bosque, ya que se emplean en actividades económicas secundarias o terciarias, tanto en la cabecera municipal como en la ciudad de San Cristóbal de Las Casas. El ingreso económico resultante permite que mucha gente pueda comprar la leña en vez de recolectarla, y en consecuencia, al disminuir la frecuencia de visita al bosque, disminuye la posibilidad de encontrar hongos.

"Esos hongos se cortaban para comer. Antes sí salía más al bosque con mi abuelita. Ya después salí a trabajar y se olvidó el hongo... antes éramos más pobres. Ahora ya no vamos por leña" (Anónimo, agosto de 2017).

Diversos autores ya han comentado la idea que existe entre muchos grupos en el mundo acerca de la recolección de hongos silvestres como una práctica asociada a la condición de pobreza de las familias campesinas (Prance, 1984; Ruan-Soto et al., 2007; Van Dijk et al., 2003). Para estos grupos, el lograr dejar atrás esta condición de pobreza, por lo regular va acompañado del olvido de los recursos silvestres y de las prácticas de aprovechamiento asociadas a este penoso pasado. Pese a esta limitación en la abundancia del recurso y a los cambios en las dinámicas económicas de algunas personas, un sector de la población continúa saliendo al bosque para pastorear sus borregos y obtener leña, teniendo la posibilidad de recolectar hongos en la temporada de lluvia. Esto prevalece sobre todo en los parajes más alejados de la cabecera municipal, con menor densidad poblacional y con mayor cantidad de cobertura boscosa. La recolecta de hongos comestibles no solamente se destina al consumo en el interior de la unidad familiar, sino también a la comercialización en conjunto con otras plantas silvestres, ya sea en los mercados de San Cristóbal de Las Casas o la cabecera municipal de Chamula, o incluso de casa en casa por encargo. Tales prácticas han permitido que incluso las personas que ya no recolectan hongos, continúen comiendo diferentes especies de hongos silvestres.

"Camino solita buscando hasta que me canso. Todo los hongos que recolecto los vendo y me piden más, algunos los como yo. Cuando voy a buscar a los hongos además voy a buscar las plantas que hago ramos para los baños de los niños. Mucha gente en Jomaló van por hongos, hombres y mujeres, mucha gente va a buscar yuyos al monte" (Anónimo, junio de 2017).

El comercio de hongos silvestres es sin duda una actividad practicada de manera frecuente por muchas comunidades micófagas en el país, ya que deja beneficios económicos relativamente altos a los recolectores (Montoya et al., 2001; Ruan-Soto et al., 2006). Sin embargo, a diferencia del centro del país donde el número de especies comercializadas puede ascender a varias decenas, en Chamula no se encontró la comercialización de muchas especies. Las principales son el -yuy- (Amanita hayalyuy) y el -k'antsu- (A. jacksonii), las cuales son las especies más ofertadas y demandadas. Los esporomas de estas especies se ofertan tanto en estado maduro como inmaduro. Cuando los yuyos se encuentran en esta fase de desarrollo, los recolectores tienen la precaución de retirarles una parte del velo universal para que los compradores puedan verificar la identidad de la especie. También se registró la comercialización de -chechev- (Armillaria mellea) y de -moni'(Agaricus spp.), aunque en mucho menor escala. En años recientes, las vendedoras de hongos prefieren entregar el producto de casa en casa por encargo, ya 
que temen que las autoridades sanitarias o municipales acudan al mercado y decomisen sus hongos, esto debido a las políticas de prohibición que se han implementado como consecuencia de algunos sucesos de intoxicación ocurridos en la última década (Ruan-Soto et al., 2012).

\section{Conocimientos etnomicológicos}

En general la gente tanto recolectora como no recolectora, sabe con precisión que los hongos aparecen durante la época de lluvias. De manera específica el -yuy- (Amanita hayalyuy) y el -k'antsu-(A. jacksonii) se encuentran desde fines del mes de mayo y durante todo junio e inclusive parte de julio, asociándose su presencia a la fiesta de San Juan, el 24 de junio. Otras especies como el -moni'- (Agaricus spp.) aparecen antes, a finales de abril con las primeras lluvias. Por el contrario, especies como el -chechev San Andrés- (Armillaria mellea) aparece con las últimas lluvias en los meses de octubre y noviembre. Finalmente, especies como el -taj chuch- (Neolentinus lepideus) se encuentran antes de que comience la época de lluvias, en los meses de marzo o abril.

La manera de preparar los hongos no es muy variada y depende en gran parte de la cantidad de especímenes que se encuentren y/o se compren. Si se tiene una recolecta abundante, los hongos se preparan en caldos o en guisos tradicionales como el -puch' emo el -vok ich-, preparados a base de maíz. Si se encuentran pocos ejemplares, solamente se asan en el comal. En pueblos micófilos existe un conocimiento culinario sumamente extenso sobre cómo preparar diferentes especies de hongos silvestres, evidenciado su importancia y su lugar como parte de sus tradiciones (Kurczyn, 2002; Valadez et al., 2011). Por lo general, no se mezclan diferentes especies de hongos al momento de hacer los guisados. Esta práctica es altamente recomendada en los esquemas de prevención de la micología académica y las instituciones de salud para tener mayor certeza de la identidad taxonómica del hongo que pudiera causar una eventual intoxicación (Lincoff y Mitchel, 1977).

Entre los entrevistados, las especies comestibles y en general aquellas que tienen alguna utilidad tienen nombre en tsotsil, como es descrito a detalle en los trabajos etnomicológicos desarrollados con anterioridad con los tsotsiles de Los Altos de Chiapas (Shepard et al., 2008). Sin embargo en estos mismos textos y en el trabajo de campo se pudo observar que, salvo algunas excepciones, las especies tóxicas por lo general no son nombradas. Las personas que sí mencionan nombres para estas especies lo hacen utilizando el nombre de la especie comestible a la que se parece y agregando el adjetivo jmilvanej- que significa asesino, haciendo evidente la condición tóxica de éstas. Ejemplo de ello se menciona a -chechev jmilvanej- (Galerina marginata) o -Sekub t'ul jmilvanej- (Suillelus luridus) haciendo referencia a sus contrapartes comestibles, chechev (Armillaria mellea) y Sekub t'ul (Boletus spp.). Asimismo, en lo que respecta al conocimiento etnoecológico que se tiene sobre estas especies tóxicas, los entrevistados refieren de manera muy general, que aparecen en los mismos meses que sus -compañeros comestibles- y en los mismos hábitats. Estas respuestas evidencian la falta de finura en el conocimiento sobre especies tóxicas la cual sí expresan para los hongos comestibles. Este mismo patrón se ha observado entre pueblos nahuas y mestizos del estado de Tlaxcala (Ramírez-Terrazo, 2017). Para Hunn (1982) solamente una parte de las discontinuidades naturales son reconocidas en las clasificaciones etnobiológicas: aquellas más próximas a la vida humana y que generen cierto interés. El resto de las entidades son reconocidas solamente en términos muy generales. Para los tsotsiles de Chamula, las especies tóxicas caen en este grupo de organismos a los cuales se les reconoce solo a partir de su diferencia con las especies comestibles.

Amanita muscaria es una excepción a este patrón (Figura 2). Esta especie recibe el nombre de -yuy chauk- que significa yuyo de rayo, por la relación estrecha que guarda con este elemento atmosférico; a su vez estos fenómenos guardan relación con el agua y las serpientes (De la Garza, 2003). En Chamula las serpientes, entre otros animales, son los guardianes del bosque acompañantes de los -anjeletik-, seres divinos que cuidan de los cerros y administran ciertos elementos de naturaleza fría como el agua, el viento, las cuevas, por mencionar algunos (Page, 2005). A través de los rayos y con las primeras lluvias, los -anjeletik- envían los hongos a la tierra. Los entrevistados mencionan que en el sitio donde cae un rayo, aparece un -yuy chauk- para que las serpientes adquieran su veneno al comerlos. La importancia de este hongo quizá radique en esta función que cumple en el universo tsotsil, y una consecuencia es su alta frecuencia de reconocimiento entre las personas, a diferencia del resto de las especies tóxicas. 
Estrategias para la identificación de las especies comestibles

La poca atención a las especies tóxicas también se ve reflejada en las estrategias que tiene la gente para distinguir las especies que se pueden comer de las que son tóxicas. El conocimiento para ello se centra en las especies comestibles y no en las tóxicas.

"Sí se reconocer los hongos que se comen. Los tóxicos no los conozco bien, pero si no los conozco, no los recolecto" (Anónimo, mayo de 2017).

La estrategia más generalizada para distinguir las especies es reconocer a la perfección la morfología de los hongos comestibles y contrastar las características observadas de los ejemplares que se encuentran en el bosque con ese modelo teórico. Particularmente en el caso de los -yuy- (Amanita hayalyuy) existe la norma generalizada entre las personas que para ser reconocido como tal, los ejemplares deben de presentar -svex- o calzón (el anillo colgante sobre su estípite). Del mismo modo, el -chechev- (Armillaria mellea) y el -ikal yuy- (Amanita vaginata) deben de tener -svex- para ser considerados como comestibles. En este sentido, se observa la regla "si tiene svex se come y si no lo tiene es veneno". En el caso de recolectar -sekum t'ul- (Boletus spp.) el ejemplar no se debe de manchar de negro o verde al cortarlo o manipularlo para ser reconocido como comestible. Aunque estas normas fueron las más mencionadas, en el trabajo de campo se observó que no pocos entrevistados tenían conocimientos contrarios, señalando que los -sekum t'ul- comestibles son aquellos que se manchan de negro al partirlos y que incluso existen yuyos tóxicos que también tienen -svex-. En general, todos los pueblos micófagos en el mundo han desarrollado distintas estrategias y criterios que con el tiempo se traducen en normas que se siguen con la esperanza de evitar intoxicaciones (Ramírez-Terrazo et al., 2014). Por otro lado, desde la micología académica y las instituciones de salud, estas normas generalizadoras siempre son señaladas como imprecisas y se aconseja no seguirlas (Lincoff y Mitchel, 1977). En el caso concreto de las especies del género Amanita, la presencia de anillo no puede considerarse como un carácter que indique su comestibilidad ya que existen especies comestibles que no lo presentan (como por ejemplo Amanita vaginata) y especies tóxicas que lo presentan (como por ejemplo Amanita bisporigera). En el caso particular de los tsotsiles de Chamula, llama la atención que no existen normas generalizadas que se conozcan de manera precisa, sino criterios en ocasiones contrapuestos. Este hecho sin duda pueden ser considerado como una de las causas de las intoxicaciones entre la población de este municipio (Ruan-Soto et al., 2012).

Otra estrategia de reconocimiento que se sigue cuando existe duda de si la identidad comestible de un ejemplar recolectado, es probando un poco del hongo e identificar su sabor; si este es desagradable, picante o amargo, es un indicador de su toxicidad. Esta prueba se usa particularmente para el etnotaxón -yixim chij(Ramaria spp.) del cual señalan: "Recolectan los hongos y lo hacen siempre, recolectan y si saben amargo o picante o saben mal los tiran. Saben jik' ik' como sabor a papa verde" (Anónimo, agosto de 2017). Aun cuando esta estrategia puede ser efectiva para ciertos taxa, para otros, particularmente aquellas especies que contienen faloidinas como por ejemplo las amanitas blancas (Figura 2), esto podría ocasionar una grave intoxicación (Talamoni et al., 2006).

Otra estrategia a la que se apega la gente para evitar confusiones es recolectar solamente en los sitios que son reconocidos como propios de los hongos comestibles. Para los entrevistados si los hongos son recolectados en sitios diferentes a los usuales dónde previamente han recolectado hongos comestibles, se podría correr el riesgo de recolectar una especie tóxica: "El yuy solo sale en algunos lugares, si se encuentran en otro lado, no es yuy" (Anónimo, agosto de 2017).

El sustrato de crecimiento es otro indicador frecuentemente mencionado, evitándose la recolección de hongos que crecen sobre excremento de animales: "Esos que crecen en el estiércol de la vaca no se comen, es veneno'" (Anónimo, junio de 2017). Diversos autores (por ejemplo Lincoff y Mitchel, 1977) hacen esta misma recomendación para evitar posibles intoxicaciones involuntarias.

Por lo general, la gente solo recolecta y consume las especies que sabe que son comestibles, sin embargo también se entrevistó a personas que en ocasiones deciden recolectar y consumir especies que no reconocen con seguridad como comestibles o inclusive especies que no conocen.

"Ese [Morchella sp.] una vez lo encontramos por la carretera y nos dio curiosidad. Nunca lo había probado. Pero lo platicamos y nos arriesgaron a probarlo, sabía muy sabroso, -no nos dio miedo- pensamos, yo creo que este se come" (Anónimo, julio de 2017). 
"Si había comido hongos, pero de ese [Amanita vaginata] nunca habíamos comido, es la primera vez que tomó nuestra hermana mayor. Es la primera vez que lo comimos" (Anónimo, julio de 2017).

\section{Proceso de enseñanza-aprendizaje de la micología tsotsil}

Los conocimientos que permiten distinguir las especies que se pueden aprovechar de las que son tóxicas son enseñados a los niños en la práctica, en el día a día del campo con los mismos elementos de aprendizaje en su hábitat. Este proceso de enseñanza-aprendizaje tiene lugar, sobre todo, mientras se pastorea a los borregos y se recolecta la leña. Para la gente entrevistada, esta manera es la forma correcta en que los campesinos deben aprender los detalles de la micología tsotsil.

"Esos hongos los empecé a conocer cuando pastoreábamos con mi mama, cuando era pequeño, luego cuando iba con mi papá a rajar leña, nos enseñaba el yuyo, igual pues cortábamos la que parecía yuyo pero como él está ahí, o si ya íbamos solos pues no decían este no es, no lo traigan. Cuando yo estaba chiquito con mi mamá como pastoreábamos donde está mi mamá iba por lo borregos y de paso ahí nos enseña los hongos. Cuando ya sabía reconocer a los hongos tenía como 8 o 10 años" (Anónimo, mayo de 2017).

Por lo general, el cuidado se prolonga hasta el momento de la preparación de los hongos, donde el proceso es vigilado por un adulto conocedor para evitar, en la medida de lo posible, errores en la identificación que ocasionen intoxicaciones. Más aún si los niños acuden solos a recolectar los hongos en el bosque, práctica que es frecuente en algunos entrevistados.

"Llegando del campo, los volvía a revisar. Más si los niños fueron solos, los revisaba bien otra vez...yo mando a mis nietos y a mis hijos antes pero nunca permito que ellos cocinen solos. Antes de enviarlos, les ensañaba dónde estaban los hongos y les advertía que no los cocinaran ellos solas" (Anónimo, agosto de 2017).

Según datos de instituciones de salud, muchas de las intoxicaciones son producto de una falta de vigilancia por parte de los padres hacia los menores de edad (Chanona-Gómez, 2014), y aunque este fenómeno sí ocurre, no necesariamente es frecuente.

Entre los entrevistados existe la percepción de que cuando los niños asisten a la escuela para recibir educación formal, efectivamente aprenden diferentes temas, pero también dejan de aprender muchos de los conocimientos acerca de su medio, sus bienes natu- rales y su aprovechamiento tradicional, poniendo en riesgo la continuidad de la micología tsotsil, la cual depende en su mayoría de la oralidad y práctica empírica. En México y muchos países de Latinoamérica, en el sistema educativo formal, los temas relacionados con los conocimientos ecológicos tradicionales no son de importancia y son desplazados por un cúmulo de conocimientos descontextualizados del medio en el que se vive (Yunes-Jiménez, 2015).

"Cuando la gente va a la escuela ya no aprende todo esto de los hongos y del monte...Los jóvenes ya no saben, porque ya no llegan al monte. Antes los niños ya a los 8 años ya salían y sabían cuáles eran los que se pueden comer, salían con sus hermanas o con su mamá y les decían, salían con su cubeta y cortaban bastantes" (Anónimo, septiembre de 2017).

Aunado al factor de la educación formal como detonante de pérdida de conocimiento etnomicológico, los entrevistados también tienen la percepción de que los jóvenes ya no saben reconocer los hongos por haber cambiado las actividades económicas primarias tradicionales en las que se desarrollaban, por actividades de transformación de bienes y de prestación de servicios, que en consecuencia, los alejan del bosque y sus recursos. Sobre todo, la percepción entre los entrevistados de más edad, es que las labores del campo ya no son de interés de los jóvenes, cuando anteriormente desde temprana edad sabían reconocer las diferentes especies de hongos.

"Solo tres hijos lo conocen bien. Antes no había dinero, ahora hay jóvenes que trabajan y traen dinero. Pero ya mis hijos ya no quieren salir, ya no saben de hongos, solo quieren comprarlos" (Anónimo, septiembre de 2017).

Saynes-Vásquez et al. (2013) advierten que el cambio cultural, indicado por la actividad ocupacional y el nivel de escolaridad formal, si bien han traído un aumento en los bienes materiales de las comunidades, está negativamente asociado con la aprehensión del conocimiento etnobiológico.

\section{CONSIDERACIONES FINALES}

La recolecta segura de hongos comestibles es una actividad que demanda de las personas que la realizan un contacto continuo con los hongos en el bosque. A diferencia de otros recursos como las plantas, que se encuentra fijos y de manera más o menos perma- 
nente, los esporomas son un recurso ocasional. Esta condición de los macromicetos como recurso, hace que el aprendizaje de sus características sea un proceso más largo en el que es menester recolectar de manera continua cada año durante la temporada de lluvias para reafirmar lo aprendido. Si por algún factor, como los discutidos a lo largo de este artículo, la gente deja de acudir al bosque y en consecuencia de revisar y observar los hongos y sus características de manera continua, existe una alta probabilidad de que dichos conocimientos vayan cayendo en desuso, se olviden, o se tergiversen.

Este fenómeno acarrea consecuencias en varios sentidos. Por un lado la pérdida de conocimientos tradicionales (producto de rupturas en la cadena de trasmisión oral y práctica de dichos conocimientos) y el paulatino desuso de recursos y abandono de prácticas de aprovechamiento se debe de entender como una merma en nuestro patrimonio micocultural, perdiendo oportunidades de esquemas que permiten una mejor calidad de vida a través de aprovechamientos sustentables, justos y soberanos. Pero por otro lado, el no tener certeza de los conocimientos micológicos que permiten diferenciar las especies comestibles de las tóxicas puede traer consigo graves riesgos para la salud de los recolectores y sus familias, generando un problema de salud pública. Esto se hace evidente al observar el poco consenso que existe entre los entrevistados en las características que deben tener las especies consideradas como comestibles.

Si bien existen prácticas de recolecta y consumo adecuadas que reducen la posibilidad de intoxicaciones como no consumir las especies que no se conocen, no revolver las diferentes especies para su traslado y consumo, así como evitar aquellas con ciertos sustratos, existen otras prácticas locales que pudieran representar un riesgo, como lo es el probar los ejemplares para determinar su toxicidad y dejar solos a los niños en el proceso de recolecta y consumo de especies fúngicas. Durante los últimos años, en atención a los eventos de intoxicación que se han suscitado en distintos municipios los Altos de Chiapas, tanto instituciones ligadas a la salud como a la academia han diseñado diferentes líneas enfocadas sobre todo a la prevención de las intoxicaciones. En especial se ha hecho énfasis en mostrar a través de distintos medios de comunicación (foros, exposiciones, ferias, carteles, trípticos, por mencionar algunas estrategias) la importancia que tienen los conocimientos micológicos locales e incentivando procesos de revitalización de estos, sobre todo en la población infantil y juvenil.

Asimismo, estas campañas se han centrado en mostrar las características de los hongos tóxicos para que la población los recuerde y haga una recolecta segura. Sin embargo, como se plantea, al menos en el caso de los tsotsiles de Chamula, la población no centra su atención en estos hongos, sino en las especies comestibles. Esto sin duda es una llamada de atención a la reflexión de cómo diseñar las estrategias de prevención en materia de contenidos para que estas tengan los efectos esperados, basándose en especies de importancia cultural para las poblaciones locales.

\section{AGRADECIMIENTOS}

Se agradece al Programa de Becas Posdoctorales en la UNAM, Centro de Investigaciones Multidisciplinarias sobre Chiapas y la Frontera Sur, asesorado por el Dr. Jaime Page Pliego. Se agradece también a las personas que colaboraron en este proyecto: Manuel Pérez Gómez, Angelina Díaz Ruiz, Anastasio Hernández López, Marisa Ordaz Velázquez y Mario Palma Olmos por su apoyo en el trabajo de campo. A Marisa Ordaz Velázquez revisión del manuscrito. A Mara Ximena Haro Luna por su apoyo con los sistemas de información geográfica. A la Consultoría Yaxal Na S.C. por su apoyo logístico. A la Dirección de Protección contra Riesgo Sanitario de la Secretaría de Salud de Chiapas, especialmente al Biol. Julio Aguilar Conde por todo el apoyo prestado.

\section{LITERATURA CITADA}

Alonso-Aguilar L.E., A. Montoya, A. Kong, A. Estrada-Torres, R. Garibay-Orijel, 2014. The cultural significance of wild mushrooms in San Mateo Huexoyucan, Tlaxcala, Mexico. Journal of Ethnobiology and Ethnomedicine 10:27.

Alvarado-Rodríguez, R., 2010. Conocimiento Micológico Local y micetismo: una aproximación a la etnomicología tzeltal de Kotolte, Tenejapa, Chiapas, México. Tesis de Maestría, El Colegio de la Frontera Sur, San Cristóbal de Las Casas.

Bernard, R., 1995. Research methods in anthropology. Altamira Press, Lanham.

Bernardino, H., R. Mariaca, A Nazar, J. Álvarez., A. Torres, C. Herrera, 2016. Factores socioeconómicos y tecnológicos en el uso de agroquímicos en tres sistemas agrícolas en los Altos de Chiapas, México. Interciencia 41 (6): 382-392.

Boa, E., 2005. Wild Edible Fungi: A global overview of their use and importance to people. FAO, Roma.

Breedlove, D.E., 1981. Flora of Chiapas. Part 1. Introduction to the flora of Chiapas. California Academy of Sciences, San Francisco.

Burrola-Aguilar C., O. Montiel O, R. Garibay-Orijel, L. Zizumbo-Villarreal, 2012. Conocimiento tradicional y aprovechamiento de los hongos comestibles silvestres en la región de Amanalco, Estado de México. Revista Mexicana de Micología 35: 1-16. 
Cano, E., A. Medinaceli, O. Sanabria, A. Argueta, 2016. Código de ética para la investigación, la investigación-acción y la colaboración etnocientífica en América Latina. Etnobiología 14 (suplemento I): 22-27.

Carpenter, S., J. Trappe, J. Ammirati, 1987. Observations of fungal sucession in the Mount St. Helens devastation zone 1980-1983. Canadian Journal of Botany 65(4): 716-728.

Cayuela, L., 2006. Deforestación y fragmentación de bosques tropicales montanos en los Altos de Chiapas, México. Efectos sobre la diversidad de árboles. Ecosistemas 15(3): 192-198.

Chanona-Gómez, F., 2014. Intoxicaciones por consumo de hongos en Chiapas. Salud en Chiapas 2(4): 200-202.

Cifuentes, J., M. Villegas, L. Pérez Ramírez, 1986. Hongos. In: Lot, A., F. Chang (eds.), Manual del Herbario. Consejo Nacional de la Flora de México. A.C., México D.F. Pp. 55-64.

Colfer, C., D. Sheil, D. Kaimowitz, M. Kishi, 2006. Los bosques y la salud humana en zonas tropicales: algunas conexiones importantes. Unasylva 57: 3-10.

De la Garza, M., 2003. El universo sagrado de la serpiente entre los mayas. Universidad Nacional Autónoma de México-Instituto de Investigaciones Filológicas, México D. F.

Dighton, J., 2003. Fungi in ecosystem processes. Marcel Dekker, Basel.

Eren, S.H., Y. Demirel, S. Ugurlu, I. Korkmaz, C. Aktas, F. Mutlu, K. Güven, 2010. Mushroom poisoning: retrospective analysis of 294 cases. CLINICS 65(5): 491-6.

Garibay-Orijel R., J. Caballero, A. Estrada-Torres, J. Cifuentes, 2007. Understanding cultural significance, the edible mushrooms case. Journal of Ethnobiology and Ethnomedicine 3:4.

Garibay-Orijel, R., A. Ramírez-Terrazo, M. Ordaz-Velázquez, 2012. Women care about local knowledge, experiences from ethnomycology. Journal of Ethnobiology and Ethnomedicine 8:25.

Garibay-Orijel, R., F. Ruan-Soto, 2014. Listado de los hongos silvestres consumidos como alimento tradicional en México. In: Moreno-Fuentes, A., R. Garibay-Orijel (eds.), La Etnomicología en México. Estado del arte. Red de Etnoecología y Patrimonio Biocultural (CONACYT)-Universidad Autónoma del Estado de Hidalgo-Instituto de Biología UNAM-Sociedad Mexicana de Micología-Asociación Etnobiológica Mexicana A.C.-Grupo Interdisciplinario para el Desarrollo de la Etnomicología en México-Sociedad Latinoamericana de Etnobiología, México D.F. Pp: 91-112.

Gómez-Reyes, V.M., 2014. Micocenosis del Parque Nacional Barranca del Cupatitzio, México. Tesis doctoral. Universidad de León, León.

Graeme, K., 2014. Mycetism: A review of the recent literature. Journal of Medical Toxicology. 10: 173-189.

Härkönen, M., 1998. Uses of mushrooms by finns and karelians. International Journal of Circumpolar Health 57: 40-55.

Härkönen, M., T. Saarimäki, L. Mwasumbi, T. Niemelä, 1993. Collection of the tanzanian mushroom heritage as a form of developmental cooperation between the univertsities of Helsinki and Dar es Salaam. Aquilo Ser. Botanica 31: 99-105.

Hunn, E., 1982. The utilitarian factor in folk biological classification. American Anthropologist 84: 830-47.
INAFED, 2005. Estado de Chiapas. Enciclopedia de los Municipios de México. Disponible en http://www.e-local.gob.mx/work/templates/enciclo/chiapas/medi.htm [consultado el 29 de noviembre de 2017].

INEGI, 2015. Principales resultados de la Encuesta Intercensal, 2015: Chiapas. INEGI, Aguascalientes.

Kurczyn, S., 2002. El festín de los hongos, XVIII Festival del Centro Histórico de la Ciudad de México. Origina, México D.F.

Lampman, A., 2007. Etnomycology: Medicinal and edible mushrooms of Tzeltal Maya of Chiapas Mexico. International Journal of Medicinal Mushrooms 9: 1-5.

Lincoff, G., D.H. Mitchel, 1977. Toxic and hallucinogenic mushroom poisoning. Van Nostrand Reinhold Company, NewYork.

Mariaca, R., J. Pérez, N. León, A. López, 2007. La milpa tsotsil de los Altos de Chiapas y sus recursos genéticos. ECOSUR-UNICH. San Cristóbal de Las Casas.

Mariaca, R., L.C. Silva, C.A. Castaños, 2001. Proceso de recolección y comercialización de hongos comestibles silvestres en el Valle de Toluca, México. Ciencia Ergo Sum 8(1): 30-40.

Montoya, A., A. Estrada-Torres, A. Kong, L. Juárez-Sánchez, 2001. Commercialization of wild mushrooms during market days of Tlaxcala, Mexico. Micologia Aplicada International 13(1): 31-41.

Montoya A., C. Méndez-Espinoza, R. Flores-Rivera, A. Kong, A. Estrada-Torres, 2007. Hongos tóxicos de Tlaxcala. Libro técnico No 2. Instituto de Investigaciones Forestales Agrícolas y Pecuarias-Universidad Autónoma de Tlaxcala, México D.F.

Montoya A., E. Torres-García, A. Kong, A. Estrada-Torres, J. CabaIlero, 2012. Gender differences and regionalization of the cultural significance of wild mushrooms around La Malinche volcano, Tlaxcala, México. Mycologia. 104(4): 826-834.

Page, J., 2005. El mandato de los dioses. Etnomedicina entre los tzotziles de Chamula y Chenalhó, Chiapas. PROIMSE-UNAM, México D.F.

Perezgrovas, R., H. Castro, 2000. El borrego Chiapas y el sistema tradicional de manejo de ovinos entre las pastoras tzotziles. Archivos de Zootecnia 49: 391-403.

Prance, G.T., 1984. The use of edible fungi by amazonian indians. In: Prance G.T., M. Kallunki (eds.), Ethnobotany in the neotropics. NY Botanical Garden Publication, New York. Pp: 127-139.

Ramírez-Terrazo, A., 2017. Importancia cultural de los hongos no comestibles en dos comunidades de las faldas del volcán La Malintzi, Tlaxcala. Tesis de maestría, Instituto de Biología, Universidad Nacional Autónoma de México, Ciudad de México.

Ramírez-Terrazo, A., A. Montoya, J. Caballero, 2014. Una mirada al conocimiento tradicional sobre los hongos tóxicos en México. In: Moreno-Fuentes, A., R. Garibay-Orijel (eds.), La Etnomicología en México. Estado del arte. Red de Etnoecología y Patrimonio Biocultural (CONACYT)-Universidad Autónoma del Estado de Hidalgo-Instituto de Biología UNAM-Sociedad Mexicana de Micología-Asociación Etnobiológica Mexicana A.C.-Grupo Interdisciplinario para el Desarrollo de la Etnomicología en México-Sociedad Latinoamericana de Etnobiología, México D.F. Pp: 116-145.

Rapoport, E.H., A. Ladio, 1999. Los bosques andino patagónicos como fuentes de alimento. Bosque 20(2): 55-64. 
Ruan-Soto, F., R. Garibay-Orijel, J. Cifuentes, 2006. Process and dynamics of traditional selling wild edible mushrooms in tropical Mexico. Journal of Ethnobiology and Ethnomedicine 2:3.

Ruan-Soto, F., R. Mariaca, J. Cifuentes, F. Limón, L. Pérez-Ramírez, S. Sierra-Galván, 2007. Nomenclatura, clasificación y percepciones locales acerca de los hongos en dos comunidades de la selva lacandona, Chiapas, México. Etnobiologia 5: 1-20.

Ruan-Soto, F., R. Mariaca, R. Alvarado, 2012. Intoxicaciones mortales por consumo de hongos: una cadena de errores. Ecofronteras 44: 12-14.

Ruan-Soto, F., W. García-Santiago, 2013. Uso de los Hongos macroscópicos: estado actual y perspectivas. In: CONABIO (ed.), La biodiversidad en Chiapas: Estudio de estado. CONABIO-Gobierno del Estado de Chiapas, Ciudad de México. Pp. 243-258.

Ruan-Soto, F., J. Caballero, C. Martorell, J. Cifuentes, A. R. González-Esquina, R. Garibay-Orijel, 2013. Evaluation of the degree of mycophilia-mycophobia among highland and lowland inhabitants from Chiapas, Mexico. Journal of Ethnobiology and Ethnomedicine 9:38.

Ruiz-Sánchez, D., J. Tay-Zavala, J. T. Sánchez-Vega, H. Martínez-Garcíam, 1999. Los micetismos y su relevancia en la medicina. Revista Iberoamericana de Micologia 16: 121-129.

Sandoval, C., 2002. Investigación Cualitativa. ICFES, Bogota.

Saynes-Vásquez, A., J. Caballero, J. Meave, F Chiang, 2013. Cultural change and loss of ethnoecological knowledge among the Isthmus Zapotecs of Mexico. Journal of Ethnobiology and Ethnomedicine 9:40.

Sheppard, G. H., D. Arora, A. Lampman, 2008. The Grace of the Flood: Classification and use of wild mushrooms among the highland Maya of Chiapas. Economic Botany 62(3): 437-470.

Soares, D., 2006. Género, leña y sostenibilidad: el caso de una comunidad en los Altos de Chiapas. Economía, Sociedad y Territorio 6(21): 151-175.
Talamoni, M., S. Cabrerizo, C. Cari, M. Díaz, M. Ortiz, I. Sager, 2006. Intoxicación por Amanita phalloides, diagnóstico y tratamiento. Archivos Argentinos de Pediatría 104(4): 372-374.

Turner, N.J., A.F. Szczawinski, 1997. Common poisonous plants and mushrooms of North America. Timber press, Portland.

Valadez, R., A. Moreno-Fuentes, G. Gómez, 2011. Cujtlacochi, El Huitlacoche. Universidad Nacional Autónoma de México e Instituto de Investigaciones Antropológicas, México D.F.

Valencia, I., 2006. Uso tradicional de los hongos silvestres en San Pedro Nexapa, Estado de México. Tesis de licenciatura, Facultad de Ciencias, Universidad Nacional Autónoma de México, México D.F.

Van Dijk, H., N. Onguene, T.W. Kuyper, 2003. Knowledge and utilization of edible mushrooms by local populations of the rain forest of South Cameroon. AMBIO 32(1): 19-23.

Vasco-Palacios, A.M., S.C. Suaza, M. Castaño-Betancur, A.E. Franco-Molano, 2008. Conocimiento etnoecólogico de los hongos entre los indígenas uitoto, muinane y andoke de la Amazonía Colombiana. Acta Amazónica 38(1): 17-30.

Vázquez, M., 2015. Estudio de las comunidades fúngicas en un suelo del SO bonaerense tratado con glifosato y 2,4-D. Tesis doctoral, Universidad Nacional del Sur, Bahía Blanca.

Vilchis, G., 2015. Centro de Información Toxicológica. Salud en Chiapas 3(2): 106-109.

Yunes-Jiménez, L., 2015. La niñez y las aves de Playón de La Gloria y Tziscao, Chiapas: una aproximación didáctica a su uso y conocimiento. Tesis de maestría, El Colegio de la Frontera Sur, San Cristóbal de Las Casas. 